\title{
Effect of Far-Field Boundary Conditions on Boundary-Layer Transition
}

\author{
Fabio P. Bertolotti ${ }^{\dagger}$ \\ Institut für Strömungsmechanik, DLR, 37073 Göttingen, Germany \\ and \\ Ronald D. Joslin \\ Fluid Mechanics and Acoustics Division \\ NASA Langley Research Center, Hampton, VA 23681
}

\begin{abstract}
The effect of far-field boundary conditions on the evolution of a finiteamplitude two-dimensional wave in the Blasius boundary layer is assessed. With the use of the parabolized stability equations (PSE) theory for the numerical computations, either asymptotic, Dirichlet, Neumann or mixed boundary conditions are imposed at various distances from the wall. The results indicate that asymptotic and mixed boundary conditions yield the most accurate mean-flow distortion and unsteady instability modes in comparison with the results obtained with either Dirichlet or Neumann conditions.
\end{abstract}

\footnotetext{
$\dagger$ This research was supported by the National Aeronautics and Space Administration under NASA Contract No. NAS1-19480 while the author was in residence at the Institute for Computer Applications in Science and Engineering (ICASE), NASA Langley Research Center, Hampton, VA 23681.
} 


\section{INTRODUCTION}

In a direct numerical simulation (DNS) of spatially growing disturbances in boundary-layer flows, the infinite domain in the streamwise direction $x$ must be truncated to a finite length, while the semi-infinite extent in the plate-normal direction y may or may not be truncated. In past simulations, either a truncated domain in $y$ [1-3] has been used or the semi-infinite domain has been mapped to a finite one with a change of variables [4-7].

The physical problem unequivocally prescribes the boundary conditions at $y \rightarrow \infty$. Asymptotic boundary conditions can be implemented when the domain is truncated at a location $y$ where the mean-flow has reached a constant value. At higher $y$ locations the asymptotic boundary conditions can be approximated rather well by mixed boundary conditions (i.e. involving the function and its derivative and sometimes called Robin conditions). Sim-

pler conditions such as homogeneous Neumann, or Dirichlet conditions can also be used; however, strictly speaking, these conditions are incorrect. The question is whether the loss of accuracy due to these more easily implemented but approximate boundary conditions is acceptable.

Here, we look at the effect of the far-field boundary conditions on the evolution of a finite-amplitude two-dimensional wave in the Blasius boundary layer. We select either asymptotic, mixed, homogeneous Dirichlet, or homogeneous Neumann conditions and impose these conditions at various distances $y_{\max }$ from the wall. For this study, we employ the parabolized stability equations (PSE) to take advantage of the low computational cost (e.g., typically 15 min on a workstation for a fully nonlinear two-dimensional calculation). 
The frequency, the streamwise starting location $x_{0}$, and the initial amplitude of the Tollmien-Schlichting (TS) wave used here were previously used by Bertolotti, Herbert, and Spalart [8] in a comparison of PSE and DNS results. The DNS code of Spalart mapped the infinite domain in $y$ to a finite domain via a mapping, which enforced the correct boundary condition at infinity and avoided the approximations caused by a truncated domain. Comparisons of results between PSE and DNS showed excellent agreement for all modes, including the mean-flow distortion [9].

In the comparison between DNS and PSE results by Joslin, Streett, and Chang [10], a discrepancy was found in the mean-flow distortion component. This discrepancy was attributed to the different far-field boundary conditions imposed in the two codes; the DNS code used homogeneous Dirichlet conditions, and the PSE code used homogeneous Dirichlet conditions for all unsteady modes and a homogeneous Neumann condition for the steady mean-flow distortion term. This discrepancy motivated the current study.

\section{GOVERNING EQUATIONS}

The reference length is $\delta\left(x_{0}\right)=\sqrt{\nu x_{0} / U_{\infty}}$, which is defined at the streamwise location $x_{0}$. The corresponding Reynolds number at $x_{0}$ is $R_{0}=$ $U_{\infty} \delta\left(x_{0}\right) / \nu=400$. The nondimensional frequency of the two-dimensional TS wave is $F=2 \times 10^{6} \pi f \nu / U_{\infty}^{2}=86$, which yields $\omega=0.0344$.

The PSE equations used in this work are described in references 8 and 9. The incompressible disturbance equations are reduced to two variables (the $u$ and $v$ components of velocity) by taking the curl of the NavierStokes equations, eliminating the $w$ velocity component with the conti- 
nuity equation, and reducing the three governing equations to two equations. The disturbance field is expanded in a six-term Fourier series in time, $0, \omega t, 2 \omega t, \ldots, k \omega t, \ldots, 5 \omega t$, which leads to complex Fourier coefficients $\hat{u}_{\mathrm{k}}$ and $\hat{v}_{\mathrm{k}}$ for the velocity components. These equations take the form,

$$
\left[\mathbf{L}+\frac{d a_{\mathrm{k}}}{d x} \mathbf{N}\right] \mathbf{q}_{\mathrm{k}}+\mathbf{M} \frac{d \mathbf{q}_{\mathrm{k}}}{d x}=R_{\mathrm{k}} \quad \mathrm{k}=0,1,2, \ldots, 5
$$

where $\mathbf{q}_{\mathrm{k}}=\left\{\hat{u}_{\mathrm{k}}, \hat{v}_{\mathrm{k}}\right\}$ is the vector of profile functions, $a_{\mathrm{k}}$ is the complex wavenumber for mode $\mathrm{k}$ composed of a real part $\gamma_{\mathrm{k}}$ describing the growth rate and an imaginary part $k \alpha$ describing the wavenumber, the operators $\mathbf{L}, \mathbf{M}, \mathbf{N}$ depend on $a_{\mathrm{k}}$ and frequency $\mathrm{k} \omega$, and contain derivatives only in $\mathrm{y}$. The operator $\mathbf{L}$ contains the Orr-Sommerfeld and Squire operators, which are well known in the parallel-flow stability theory. The right-hand-side term $R_{\mathrm{k}}$ is the convolution term stemming from the nonlinear products.

Introducing the finite difference form $d \mathbf{q}_{\mathrm{k}} / d x \rightarrow\left(\mathbf{q}_{\mathrm{k}}-\mathbf{q}_{\mathrm{k}}^{\text {old }}\right) / d x$ and $d a_{\mathrm{k}} / d x \rightarrow\left(a_{\mathrm{k}}-a_{\mathrm{k}}^{o l d}\right) / d x$ into equation (1) yields

$$
\left[\mathbf{L}+\frac{a_{\mathrm{k}}-a_{\mathrm{k}}^{\text {old }}}{d x} \mathbf{N}+\frac{1}{d x} \mathbf{M}\right] \mathbf{q}_{\mathrm{k}}=R_{\mathrm{k}}+\frac{1}{d x} \mathbf{M} \mathbf{q}_{\mathrm{k}}^{\text {old }} . \quad \mathrm{k}=0,1,2, \ldots, 5
$$

These coupled set of equations can be solved by marching in $x$.

The physical boundary conditions at $y \rightarrow \infty$ impose vanishing $\hat{u}_{\mathrm{k}}$ and $\hat{v}_{\mathrm{k}}$ velocities for the unsteady modes $(\mathrm{k}>0)$ and vanishing $\hat{u}_{0}$ and constant $\hat{v}_{0}$ velocities for the steady mode $(\mathrm{k}=0)$. A finite $\hat{v}_{0}$ allows for changes in the displacement thickness as the flow transitions to turbulence. This condition becomes $\hat{v}_{0}=0$ in flows over bodies with a bounded streamwise extent.

Outside the boundary layer, the operators $\mathbf{L}, \mathbf{M}, \mathbf{N}$ have constant coefficients, and the solution decays exponentially. When the computational 
domain is truncated in this region, boundary conditions can be imposed that yield the exact solution at all interior points. These boundary conditions have been presented by Keller [11] for the Orr-Sommerfeld operator, and are extended here to the PSE equation. The basic idea is to require that the solution has a null projection onto the subspace spanned by the exponentially growing eigensolutions of the operator on the left-hand side of equation (2). These eigensolutions are evaluated when equation (2) is re-written as a first order system. In the present formulation the highest derivative in $y$ of $\hat{u}$ is 2 , and of $\hat{v}$ is 4 , hence we introduce the vector $\mathbf{x}_{\mathrm{k}}=\left\{\hat{u}_{\mathrm{k}}, \hat{u}_{\mathrm{k}}^{\prime}, \hat{v}_{\mathrm{k}}, \hat{v}_{\mathrm{k}}^{\prime}, \hat{v}_{\mathrm{k}}^{\prime \prime}, \hat{v}_{\mathrm{k}}^{\prime \prime \prime}\right\}$ were the prime denotes differentiation w.r.t. $y$, and re-write (2) as

$$
\mathbf{A} \mathbf{x}_{\mathrm{k}}+\mathbf{B} \frac{d \mathbf{x}_{\mathrm{k}}}{d y}=\mathbf{r}_{\mathrm{k}}(y)
$$

where the matrices $\mathbf{A}$ and $\mathbf{B}$ depend on $\mathrm{k}$ and contain, in addition to the information in equation (2), the relations $d\left(\hat{u}_{\mathrm{k}}\right) / d y=\hat{u}_{\mathrm{k}}^{\prime}, d\left(\hat{v}_{\mathrm{k}}\right) / d y=\hat{v}_{\mathrm{k}}^{\prime}$, $d\left(\hat{v}_{\mathrm{k}}^{\prime}\right) / d y=\hat{v}_{\mathrm{k}}^{\prime \prime}$, and $d\left(\hat{v}_{\mathrm{k}}^{\prime \prime}\right) / d y=\hat{v}_{\mathrm{k}}^{\prime \prime \prime}$. We then compute, for each mode $k$, the eigensolutions $\left\{\lambda_{\mathbf{i}}, \mathbf{e}_{\mathbf{i}}\right\}$ that solve $\left[\mathbf{A}^{T}-\lambda_{\mathbf{i}} \mathbf{B}^{T}\right] \mathbf{e}_{\mathbf{i}}=0$, where $T$ denotes transpose, and relabel these eigensolutions so that $\lambda_{1}, \lambda_{2}$ and $\lambda_{3}$ have a negative real part. The requirement of zero projection onto the growing eigenmodes yields the following asymptotic boundary conditions,

$$
\mathbf{x}_{\mathrm{k}} \cdot \mathbf{B}^{T} \mathbf{e}_{\mathbf{i}}=\mathbf{c}_{\mathrm{k}} \cdot \mathbf{e}_{\mathbf{i}} \quad \mathrm{i}=1,2,3
$$

where

$$
\mathbf{c}_{\mathrm{k}}=\left\{\frac{b_{1}}{\left(c_{1}+\lambda_{\mathbf{i}}\right)}, \frac{b_{2}}{\left(c_{2}+\lambda_{\mathbf{i}}\right)}, \ldots\right\}
$$

is obtained by approximating each component of the forcing $\mathbf{r}_{\mathrm{k}}$ in the neighborhood of the boundary by a function of the form $b e^{c y}$, and the dot product in equation (4) is defined as $\mathbf{a} \cdot \mathbf{b}=\sum a_{\mathbf{i}} b_{\mathbf{i}}$. 
The spatial DNS code solves the disturbance form of the full NavierStokes equations with high-order finite- and compact-difference methods and spectral methods. Homogeneous Dirichlet conditions are imposed in the farfield and at the wall, inflow conditions consist of the Blasius and eigenfunctions provided by linear stability theory, and the buffer domain technique [12] is used for the streamwise outflow condition. Refer to references 13 for a

discussion of accuracy issues with grid refinement and outflow buffer domain treatment.

\section{RESULTS}

Results were obtained for cases with the upper boundary placed at $y_{\max }=15,20,30,45,60,90$, and 130 . For each of these cases, computations were made that employed the following conditions for modes $\mathrm{k}=0,1,2,3,4,5:$

Dirichlet conditions:

$$
\hat{u}_{\mathrm{k}}=0, \quad \hat{v}_{\mathrm{k}}=0, \quad \frac{\partial \hat{v}_{\mathrm{k}}}{\partial y}=0
$$

Neumann conditions:

$$
\frac{\partial \hat{u}_{\mathrm{k}}}{\partial y}=0, \quad \frac{\partial \hat{v}_{\mathrm{k}}}{\partial y}=0, \quad \frac{\partial^{2} \hat{v}_{\mathrm{k}}}{\partial y^{2}}=0
$$

Mixed conditions:

$$
\frac{\partial \hat{u}_{\mathrm{k}}}{\partial y}+a_{\mathrm{k}} \hat{u}_{\mathrm{k}}=0, \quad \frac{\partial \hat{v}_{\mathrm{k}}}{\partial y}+a_{\mathrm{k}} \hat{v}_{\mathrm{k}}=0, \quad \frac{\partial^{2} \hat{v}_{\mathrm{k}}}{\partial y^{2}}+a_{\mathrm{k}} \frac{\partial \hat{v}_{\mathrm{k}}}{\partial y}=0
$$

Asymptotic conditions:

$$
\mathbf{x}_{\mathrm{k}} \cdot \mathbf{B}^{T} \mathbf{e}_{\mathbf{i}}=\mathbf{c}_{\mathrm{k}} \cdot \mathbf{e}_{\mathbf{i}} \quad \mathrm{i}=1,2,3
$$


The conditions for the highest derivative of $\hat{v}$ with respect to $y$ are derived from the continuity equation. The mixed boundary conditions and the asymptotic boundary conditions are altered for the mean-flow distortion term (i.e. $k=0$ ), to the form discussed above, namely,

$$
\hat{u}_{0}=0, \quad \frac{\partial \hat{v}_{0}}{\partial y}=0, \quad \frac{\partial^{2} \hat{v}_{0}}{\partial y^{2}}=0
$$

The initial condition was composed of the single Fourier mode $\mathrm{k}=1$, with an amplitude of 0.25 percent rms. based on the maximum of the $u$ component of velocity.

High resolution in the plate-normal direction $y$ was obtained with five subdomains. In each subdomain, the $\hat{u}_{\mathrm{k}}$ and $\hat{v}_{\mathrm{k}}$ velocity components were expanded with 18 Chebyshev polynomials. The step size in $x$ was set to $\Delta x=10$.

Figure 1 shows the evolution of the disturbance amplitude based on the $u$ component of velocity for the Fourier modes $F=1, F=2$, and the steady component $F=0$ with a Reynolds number of $R=U_{\infty} \delta(x) / \nu=\sqrt{x R_{0}}$ for results calculated by both the PSE and DNS codes. Both codes enforced the Dirichlet boundary conditions (eqs. 1 ) at $y_{\max }=130$. The results agree well, which reasonably indicates the equivalence of the two procedures for the flat-plate problem.

Next, computations were conducted with PSE theory to compare the maximum amplitudes of $F=0$ and $F=1$ modes as function of far-field boundary locations. At the downstream location that corresponds to $R=$ 940, Figure 2 displays the dependence of the maximum amplitudes, based on the $u$ component of velocity, with the truncated far-field boundary location 
$y_{\max }$ for the $F=1$ and $F=0$ modes. The solid line represents results that were obtained with asymptotic boundary conditions (eqs. (9)), the dashed line represents the mixed conditions (eqs. (8)), the square symbols represent Dirichlet conditions (eqs. (6)), the triangular symbols represent Neumann conditions (eqs. (7)), and the arrows denote the results obtained by applying the physical boundary conditions at infinity (using an algebraic mapping). Note that the boundary-layer edge (99\% definition) grows from $y=5$ at $R=400$ to $y=12$ at $R=940$; therefore, the far-field boundary must be beyond $y=12$.

The results obtained with the asymptotic boundary conditions are independent of $y_{\max }$ once the mean-flow has reached a constant value (e.g. with less than a $0.01 \%$ variation). To increase accuracy, the operators in equation (3) can be evaluated with the mean-flow value at infinity, rather than at $y_{\max }$, however, for $y_{\max }<20$ the mean-flow is still varying when the boundary is reached, and, consequently, the asymptotic boundary conditions become only approximate. The dip in the solid curve in figure 2 displays this fact. The evaluation of $\mathbf{c}_{\mathrm{k}}$ in equation (4) can also affect accuracy; when the exponential fit (which is exact in the linear case) of $\mathbf{r}_{\mathrm{k}}$ is replaced by a two term Taylor series approximation of $\mathbf{r}_{\mathrm{k}}$ at $y_{\mathrm{max}}$, the calculated growth rates fall $15 \%$ short of the exact value, even for $y_{\max } \geq 20$.

The mixed boundary conditions impose the exponential decay $\exp (-a y)$ to the solution. The complex wavenumber $a$ is an eigenvalue of $\mathbf{A}^{T}-\lambda_{\mathbf{i}} \mathbf{B}^{T}$, and when the other two decaying eigensolutions have a decay rate much higher than $a$, the mixed boundary conditions become equivalent to the asymptotic boundary conditions, provided $y_{\max }$ is sufficiently large. A dif- 
ference between the dashed and solid curves in figure 2 is barely visible at $y_{\max }<35$, although such a close agreement should not always be expected. However, for $y_{\max }>35$, the mixed and asymptotic boundary conditions lead to the same solutions. Because the mixed conditions are homogeneous and have a simpler form, they are easier to implement.

The Neumann conditions yield accurate results for $y_{\max }>45$. These conditions allow for a change in the boundary-layer displacement thickness (i.e., nonzero $v$ velocity for $F=0$ at $y_{\max }$ ). Similarly, Dirichlet boundary conditions lead to accurate results for the traveling mode $F=1$ when $y_{\max }>45$. However, the steady component $F=0$ is adversely affected by the Dirichlet boundary conditions even for large values of $y_{\max }$, as indicated by the square symbols in Figure 2a. Furthermore, the $v$ component of velocity vanishes, which prevents changes from the laminar value of the boundary-layer displacement thickness. Figure 3 displays the $\hat{u}_{0}$ and $\hat{v}_{0}$ velocity components for the mean-flow distortion mode $(F=0)$ at $R=940$. Outside the boundary layer, the $\hat{v}_{0}$ velocity decreases linearly to match the zero boundary value at $y_{\max }=130$. Because the boundary-layer displacement thickness tends to increase beyond the laminar value, mass conservation forces a nonzero $\hat{u}_{0}$ component of velocity outside the boundary layer, which, in turn, creates an artificial boundary layer at $y_{\max }$. (Note that the small errors between the DNS and PSE $v_{o}$ profiles are of the order $10^{-6}$, which can be attributed to numerical errors in the DNS approach.)

All PSE calculations up to this point have been done using of 90 Chebyshev polynomials in $y$ per variable, per mode. This high resolution was chosen in order to remove resolution issues from the analysis. To assest the 
effect of the far-field resolution, we have repeated the computation using two domains, the inner one going from the wall to $y=5$ and the outer one from $y=5$ to $y=30$. A linear mapping from physical to $[-1,1]$ was used in both domains. The resolution in the lower domain was fixed at 18 polynomials per variable, and in the outer domain either 10 or 30 polynomials were employed. Table 1 below displays the maximum $u$ amplitude for the $F=0$ and $F=1$ modes at $R=940$ obtained with different boundary conditions. The exact values are $0.595 \%$ for $F=0$ and $2.843 \%$ for $F=1$. (The column labeled "High Res" displays the values shown in figure 2.)

Table 1. Modal maximums at $R=940$

\begin{tabular}{|c|c|c|c|c|c|c|}
\hline & & $\mathrm{F}=0$ & & & $\mathrm{~F}=1$ & \\
\hline BC Type & 10 & 30 & High Res & 10 & 30 & High Res \\
\hline Asymptotic & 0.605 & 0.595 & 0.596 & 2.966 & 2.840 & 2.844 \\
Mixed & 0.597 & 0.597 & 0.598 & 2.869 & 2.853 & 2.858 \\
Neumann & 0.666 & 0.684 & 0.684 & 3.709 & 3.807 & 3.812 \\
Dirichlet & 0.152 & 0.286 & 0.298 & 2.010 & 1.855 & 1.895 \\
\hline
\end{tabular}

The asymptotic, mixed, and Neumann conditions display only a small variation with change in resolution. The Dirichlet condition is more sensitive, due to the need to resolve the artificial boundary-later at the upper domain, shown in figure 3 . The largest difference between results, thus, comes from the boundary condition implemented, rather than the resolution.

\section{CONCLUSIONS}

The use of a finite domain in $y$ plus Dirichlet and Neumann boundary conditions eliminates some coding difficulties in direct Navier-Stokes simulation codes, but introduces errors. As in the case considered here, the errors are small when the truncation location $y_{\max }$ is located well into the region 
of exponential decay of the disturbances. An exception is the steady component $F=0$, which does not decay in the free stream and for which the error introduced by the use of Dirichlet conditions does not vanish as $y_{\max }$ is increased. A similar error also is expected for three-dimensional steady disturbances because they decay slowly (i.e., as in $\exp \left(-\beta^{2} y\right)$ ) in the free stream. The errors introduced in the calculation of traveling modes by either Dirichlet or Neumann conditions, on the other hand, are negligible if a truncation location $y_{\max }$ is chosen sufficiently far from the plate. In contrast, asymptotic boundary conditions and mixed boundary conditions yield accurate results when imposed beyond the $99.99 \%$ definition of the boundary layer edge. The asymptotic conditions are exact, but require a significantly greater amount of coding to implement. 


\section{REFERENCES}

1. H. Fasel, Investigation of the stability of boundary layers by a finitedifference model of the Navier-Stokes equations, J. Fluid Mech. 78, 355 (1976).

2. H. Fasel and U. Konzelmann, Non-parallel stability of a flat plate boundary layer using the complete Navier-Stokes equations, J. Fluid Mech. 221, 311 (1990).

3. M. M. Rai and P. Moin, Direct numerical simulation of transition and turbulence in a spatially evolving boundary layer, J. Comp. Phys. 109, $169(1993)$.

4. P. R. Spalart, A spectral method for external viscous flows, Contemp. Math. 28, 315 (1984).

5. T. A. Zang and M. Y. Hussaini, Numerical simulation of nonlinear interactions in channel and boundary-layer transition, Nonlinear Wave Interactions in Fluids, AMSE, AMD-Vol. 87.131 (1987).

6. E. Laurien and L. Kleiser, Numerical simulation of boundary-layer transition and transition control, J. Fluid Mech. 199, 403 (1989).

7. P. R. Spalart, R. D. Moser, and M. M. Rogers, Spectral methods for the Navier-Stokes equations with one infinite and two periodic directions, J. Comp. Phys. 96, 297 (1991).

8. F. P. Bertolotti, Th. Herbert, and P. R. Spalart, Linear and nonlinear stability of the Blasius boundary layer, J. Fluid Mech. 242, 441 (1992).

9. F. P. Bertolotti, Linear and nonlinear stability of boundary layers with streamwise varying properties, Ph.D. Thesis, The Ohio State University, (1991). 
10. R. D. Joslin, C. L. Streett, and C. L. Chang, Spatial direct numerical simulation of boundary-layer transition mechanisms: validation of PSE theory, Theort. Comput. Fluid Dynam., 4(6), 271 (1993).

11. H. B. Keller, Numerical Solution of Two-Point Boundary Value Problems, Regional conference series in applied mathematics. Society for Industrial and Applied Mathematics, 24, (1976).

12. C. L. Streett and M. G. Macaraeg, Spectral multi-domain for large-scale fluid dynamic simulations, Int. J. Appl. Numer. Math., 6, 123 (1989).

13. R. D. Joslin, C. L. Streett, and C.-L. Chang, Validation of threedimensional incompressible spatial direct numerical simulation code-A comparison with linear stability and parabolic stability equations theories for boundary-layer transition on a flat plate, NASA TP-3205, (1992). 
FIG. 1. Amplitudes of $F=0, F=1$, and $F=2$ modes from PSE (solid line) and DNS (symbols) with Dirichlet boundary conditions.

FIG. 2. Maximum amplitudes of $F=0$ and $F=1$ modes as function of $y_{m}$ for the case of asymptotic (solid line), mixed (dashed line), Neumann (triangles), and Dirichlet boundary conditions (squares), and physical boundary conditions at $y_{\max } \rightarrow \infty$ (arrow) at $R=940$.

FIG. 3. Velocity components $u$ and $v$ for the $F=0$ mode of PSE theory with asymptotic (line) and Dirichlet (dashed) boundary conditions and DNS results (symbols) at $R=940$. 


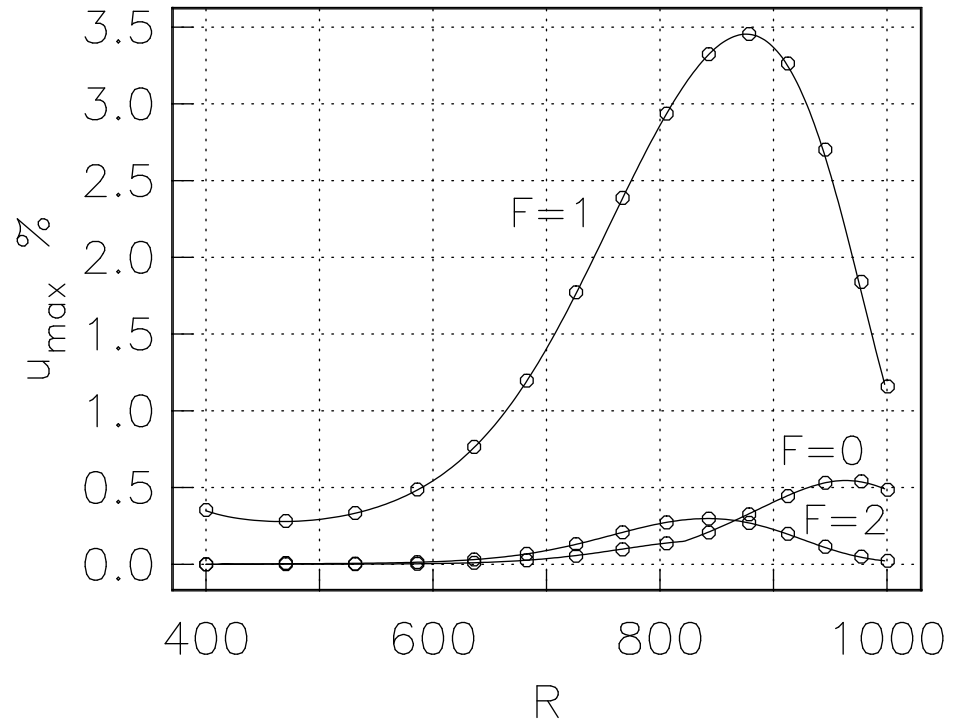

FIG. 1. Amplitudes of $F=0, F=1$, and $F=2$ modes from PSE (solid line) and DNS (symbols) with Dirichlet boundary conditions. 

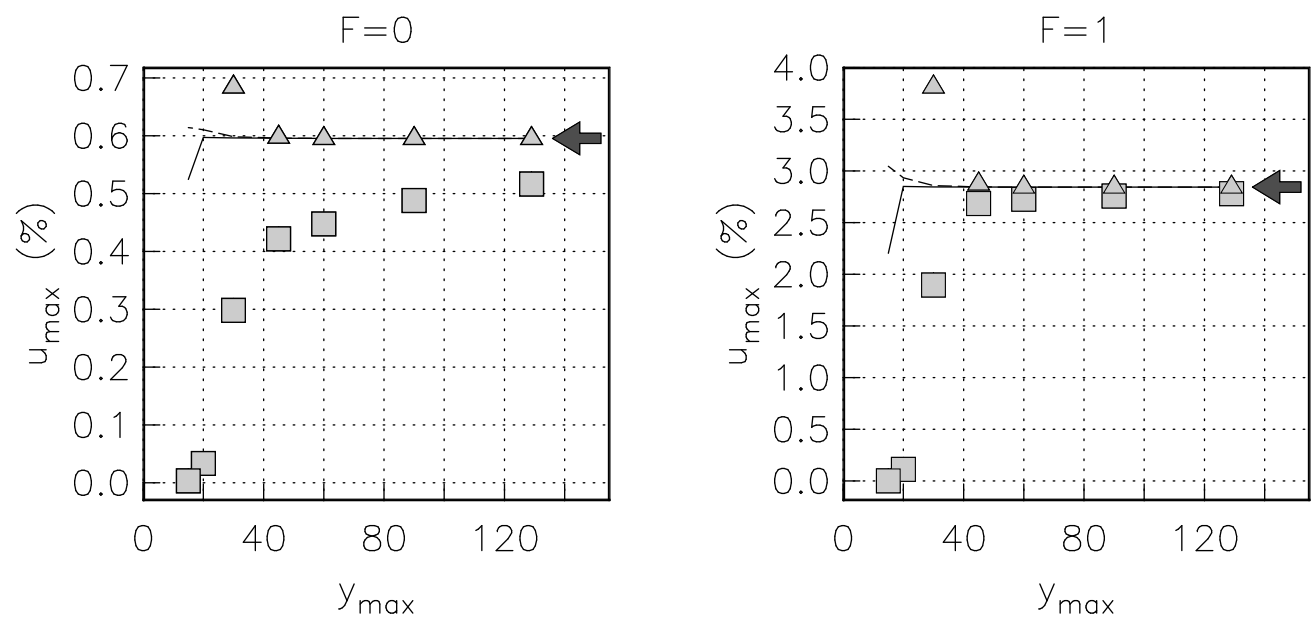

FIG. 2. Maximum amplitudes of $F=0$ and $F=1$ modes as function of $y_{m}$ for the case of asymptotic (solid line), mixed (dashed line), Neumann (triangles), and Dirichlet boundary conditions (squares), and physical boundary conditions at $y_{\max } \rightarrow \infty$ (arrow) at $R=940$. 

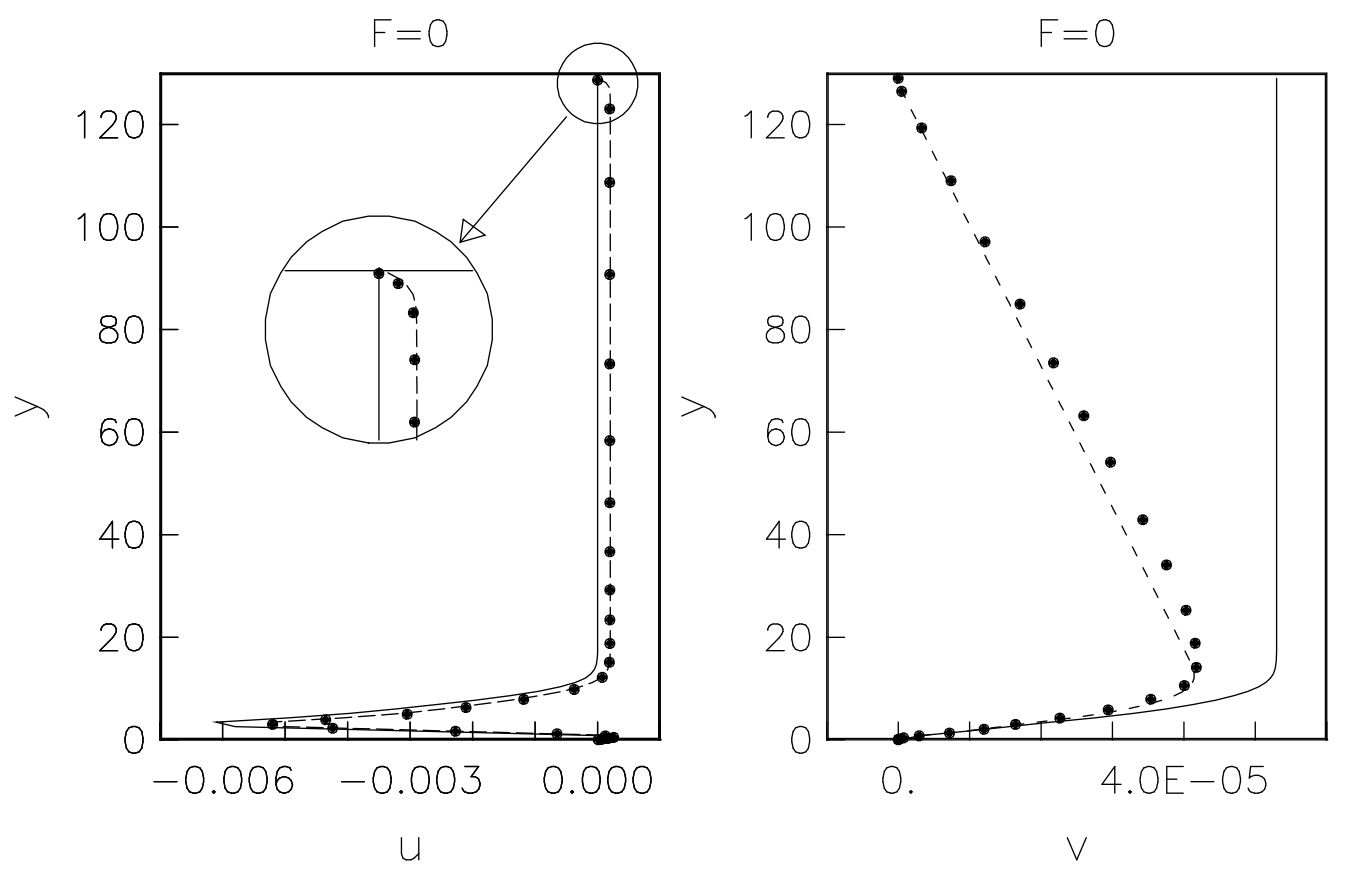

FIG. 3. Velocity components $u$ and $v$ for the $F=0$ mode of PSE theory with asymptotic (line) and Dirichlet (dashed) boundary conditions and DNS results (symbols) at $R=940$. 